$\mathrm{A} \& \mathrm{~L}$

ISSN 2709-0205

Vol 1 Issue 2 (2020)

\title{
Express diagnostics of accelerated human aging using the questionnaire
}

\author{
Anatoly V. Pisaruk, Valeri B. Shatilo, Ivanna A. Antonyuk-Scheglova, Svitlana S. Naskalova \\ State Institution “D.F. Chebotarev Institute of Gerontology NAMS of Ukraine”, 04114, Kiev \\ Correspondence: naskalov@bigmir.net
}

Received: 30.10.2020; Accepted: 11.11.2020; Published: 12.11.2020

\begin{abstract}
An express method of diagnosing accelerated aging of a person has been developed with the help of a questionnaire, which includes 15 indications of somatic aging, 12 indications of psychological aging and 10 objective indicators. The Bayesian method is used for the calculation of biological age. The accuracy of the definition for the biological age was 8.3 years. The method developed is notable for its simplicity, ease and accessibility for use in the outpatient and inpatient conditions. This method can be used for pre-selection of the patients with the risk of accelerated aging for the further in-depth studies.
\end{abstract}

Keywords: biological age; accelerated aging; indicators of aging; Bayesian formula

Diagnostics of accelerated aging is carried out in order to assess the risk of development of age-related pathology and assessment of influence of the preventive and therapeutic interventions [1-4]. Currently, gerontology usually uses the diagnostics of accelerated aging by calculating biological age (BA). The general concept of BA is based on the assumption that the individual rate of aging can vary in a significant degree [3,5]. The calculation of BA in the most of works is based on the measurement of quantitative indicators that are called biological markers of aging. These indicators should correlate highly enough with age, be available for the measurement in clinical conditions and depend not much on the current pathological conditions. A variety of indicators can be performed as biological markers: anatomical, functional, biochemical, immunological, genetic and epigenetic [69]. Currently, there is no generally accepted set of such markers. A number of studies have shown that agerelated changes in different organs and systems are developing at different velocity ("heterchronity" of aging) [5].

BA can both coincide with the chronological age, and in this case the aging process is evaluated as normal, physiological, and significantly exceed the chronological. In the latter case, aging is evaluated as accelerated. The value of the difference between biological and chronological age, which is considered to be physiological or pathological, depends on the error (inaccuracy) of the definition of BA. If this difference exceeds the value of the standard error, you can consider aging to be accelerated.

A generally accepted mathematical method of assessing of the biological age is the calculation of the multiple regression equation linking chronological age and a number of quantitative indicators. Data from a large number of practically healthy people of different ages are used to determine the coefficients of this equation. To determine the BA of the subject, a number of studies is conducted and his age is culculated using the resulting equation. If the calculated age of a person is significantly exceeded over the chronological age, a conclusion about accelerated aging is made. Many instrumental and laboratory research methods are usually used for this purpose. In the research of a large team of authors, 1,497 genes and a number of physiological and biochemical indicators, such as blood pressure, cholesterol level and blood glucose, body mass index, etc., were studied [10]. This research shows that the averaged differences between chronological age and BA is 7.8 years. 
As clinical practice shows, there is a need for simple methods that allow to carry out preliminary screening of people in order to identify the indicatiors of accelerated aging. The easiest and most publicly available of these methods is the use of the questionnaire. However, the regression analysis, that is traditionally used for this purpose, is not applicable for the data obtained by the questionnaire. This is due to the nominal character of the indicators - questionnaire questions. However, we can calculate, in that case, the frequency of premature aging symptoms in groups of relatively young and old people. The formula for the theory of probability of Bayes is used for the quantitative assessment of the informativeness of the symptoms of different pathological conditions [11, 12]. The purpose of this study was to assess the possibility of express diagnostics of accelerated aging using a specially developed questionnaire. Validation of the panel of indicators, included in this questionnaire, is carried out using the Bayes method.

\section{Materials and methods}

Based on the literary data and our own clinical experience, we have developed a questionnaire that includes 15 questions reflecting somatic changes and 12 questions that characterize the psychological changes that are distinctive for human aging. Also, the questionnaire includes 10 objective indicators that are changing during aging.

The examined people were informed that the questions are talking about slow changes that have been developing over the years. Only in this case they can be associated with the aging process. Recent changes do not refer to the indicators of aging and may be associated with temporary deterioration of health.

To collect data, online method developed by Google (so-called Google forms) was used. This method allows you to distribute the questionnaire on the Internet and get information about the answers to its questions in the form of Google Table on Google Drive. A Google form of the questionnaire, including 37 indicators of aging, was developed. This questionnaire can be downloaded to the browser at the https://forms.gle/Hh2QuERsofzM3Foi7 link. References to this questionnaire were placed in the gerontological groups of Facebook and distributed with the help of Viber among the staff of the Institute of Gerontology NAMS of Ukraine and their acquaintances. Respondents were asked to choose "Yes" or "No" for each item on the questionnaire, depending on whether they had one or another indicator of aging. We received responses from 158 people between the ages of 20 up to 79 years old. The frequency of indicators of aging in the age groups of 20-39 years old and of 60-79 years old and their diagnostic coefficients (DC) was calculated. DC indicator of aging was calculated as a decimal logarithm $(\log 10)$ ratio of frequency of the indicators in groups of 60-79 years old and of 20-39 years old, multiplied by 10. This assessment of informativeness is based on the formula of the probability theory of Bayes in the modification of E.V. Gubler. The solution of the diagnostic task by this method is in summaration of the DC of the symptoms that the patient has and the comparison of the received amount with the threshold one. In our work, we used an original approach for the calculation of BA. A linear regression equation linking age and the amount of DC was calculated. As a result, we have received the formula for calculating of the following type of $\mathrm{BA}$ : BA $=\mathrm{k} 1 \times \Sigma \mathrm{DC}+\mathrm{k} 2$ (where $\mathrm{k} 1$ and $\mathrm{k} 2$ are coefficients).

Statistical processing of the obtained data was carried out with the help of Excel 2007 and Statistica 7 programs. Standard statistical procedures, including variation and regression analyses, were used.

\section{Results and discussion}

The table demonstrates the frequencies of indicators of aging in groups of people of 20-39 and of 60-79 years old. You can see that the frequency of indicators increases with age. Differences of all indicators are reliable ( $p<0,01$ ) among the people older than 60 years old, compared to the people of 20-39 years old. For many indicators these differences are very significant - at times. According to the received frequencies the values of DC for each indicator of aging were calculated. DC characterizes the informativeness of the indicator to solve a particular diagnostic task. In this case, the task was to diagnose the rate of aging. 
Frequency of indicators of aging (\%) in different age groups and their diagnostic coefficients (DC)

\begin{tabular}{|c|c|c|c|}
\hline Age groups, years & 20-39 & $60-79$ & DC \\
\hline \multicolumn{4}{|c|}{ Somatic indicators of aging } \\
\hline You find it difficult to put on socks, shoes & 2,70 & 24,3 & 9,54 \\
\hline It became necessary afternoon sleep & 10,8 & 28,2 & 4,16 \\
\hline Movements have become slow & 1,00 & 15,8 & 11,98 \\
\hline Appeared an increased fatigue & 27,0 & 38,5 & 1,53 \\
\hline You started to sleep badly at night & 24,3 & 41,0 & 2,27 \\
\hline Appeared shortness of breath when walking fast & 16,2 & 47,2 & 4,64 \\
\hline You have started to walk slower & 5,41 & 28,9 & 7,29 \\
\hline You have begun to forget names, words & 21,62 & 55,3 & 4,08 \\
\hline Hair began to thin considerably & 27,0 & 60,5 & 3,50 \\
\hline Your hearing had worsened & 5,41 & 18,4 & 5,32 \\
\hline Your vision had worsened significantly & 18,9 & 64,9 & 5,35 \\
\hline A wobbly gait had appeared & 1,012 & 8,11 & 9,09 \\
\hline Pigment spots had appeared on the skin & 21,6 & 70,3 & 5,12 \\
\hline The numerous wrinkles had appeared on the face & 5,41 & 20,5 & 5,79 \\
\hline Dry skin had appeared on the hands & 32,4 & 64,1 & 2,96 \\
\hline \multicolumn{4}{|c|}{ Psychological indicators of aging } \\
\hline In clothes, the main thing for you became comfort, but not style & 40,6 & 53,8 & 1,22 \\
\hline Pop music no longer attracts you & 10,8 & 30,8 & 4,54 \\
\hline You had begun to fall asleep in front of the TV & 27,0 & 43,6 & 2,08 \\
\hline Social events no longer interest you & 1,01 & 7,69 & 8,86 \\
\hline You became afraid of problems & 16,2 & 42,1 & 4,14 \\
\hline
\end{tabular}




\begin{tabular}{|c|c|c|c|}
\hline You think that it used to be better & 5,41 & 51,3 & 9,77 \\
\hline You have started watching more TV & 5,56 & 35,9 & 8,10 \\
\hline Mass events had begun no longer interest you & 51,4 & 66,7 & 1,13 \\
\hline You have fewer desires & 19,4 & 34,1 & 2,45 \\
\hline You began to believe that changes are more often for the worse & 2,70 & 43,6 & 12,1 \\
\hline Now, 2-3 channels on TV became enough for you & 21,6 & 43,6 & 3,04 \\
\hline Many things have become not interesting & 16,7 & 34,21 & 3,12 \\
\hline \multicolumn{4}{|l|}{ Objective indicators } \\
\hline Body mass index is more than 25 & 27,0 & 39,5 & 1,65 \\
\hline Systolic blood pressure is more than $140 \mathrm{~mm} \mathrm{Hg}$ & 1,02 & 17,9 & 12,5 \\
\hline Pulse blood pressure is more than $60 \mathrm{~mm} \mathrm{Hg}$ & 5,56 & 35,9 & 8,10 \\
\hline Static balancing is less than 10 seconds & 8,11 & 43,6 & 7,30 \\
\hline The ratio of volume between waist and hip is more than 1 & 22,2 & 34,2 & 1,87 \\
\hline The number of removed teeth is more than 4 & 27,8 & 74,4 & 4,28 \\
\hline You wear glasses with +1 dioptry or more & 18,9 & 51,3 & 4,33 \\
\hline The pinch test is for more than 2 seconds & 16,2 & 57,9 & 5,53 \\
\hline Blood sugar is above $5,0 \mathrm{mmol} / \mathrm{L}$ & 11,1 & 30,8 & 4,42 \\
\hline Blood cholesterol is above $6,0 \mathrm{mmol} / \mathrm{L}$ & 11,1 & 38,2 & 5,37 \\
\hline
\end{tabular}

For this purpose, the DC of each of the subjects was summarized. The obtained sums were highly correlated with the chronological age of these people $(R=0,66 ; p<0,00001)$. Based on the obtained data, a regression equation was calculated, linking the chronological age of a person and the amount of his DC. The age of the person, calculated by this equation, represents his BA.

$$
\mathrm{BA}=0,2 \Sigma \not C+42,0
$$

In accordance with this formula, the values of DC indicators of aging, which the person has, are summarized and the amount is multiplied by the value of the coefficient $k 1=0,2$ and a ratio of $k 2=42,0$ is added. If the BA, calculated in this way, significantly exceeds the chronological age of the person, it can be concluded about accelerated aging.

On the figure 1 a graph of the connection of the amount of the DC and the chronological age of the surveyed people is represented. The points on the graph show the individual values of the amount scored of DC for each of the surveyed people and their chronological age. 


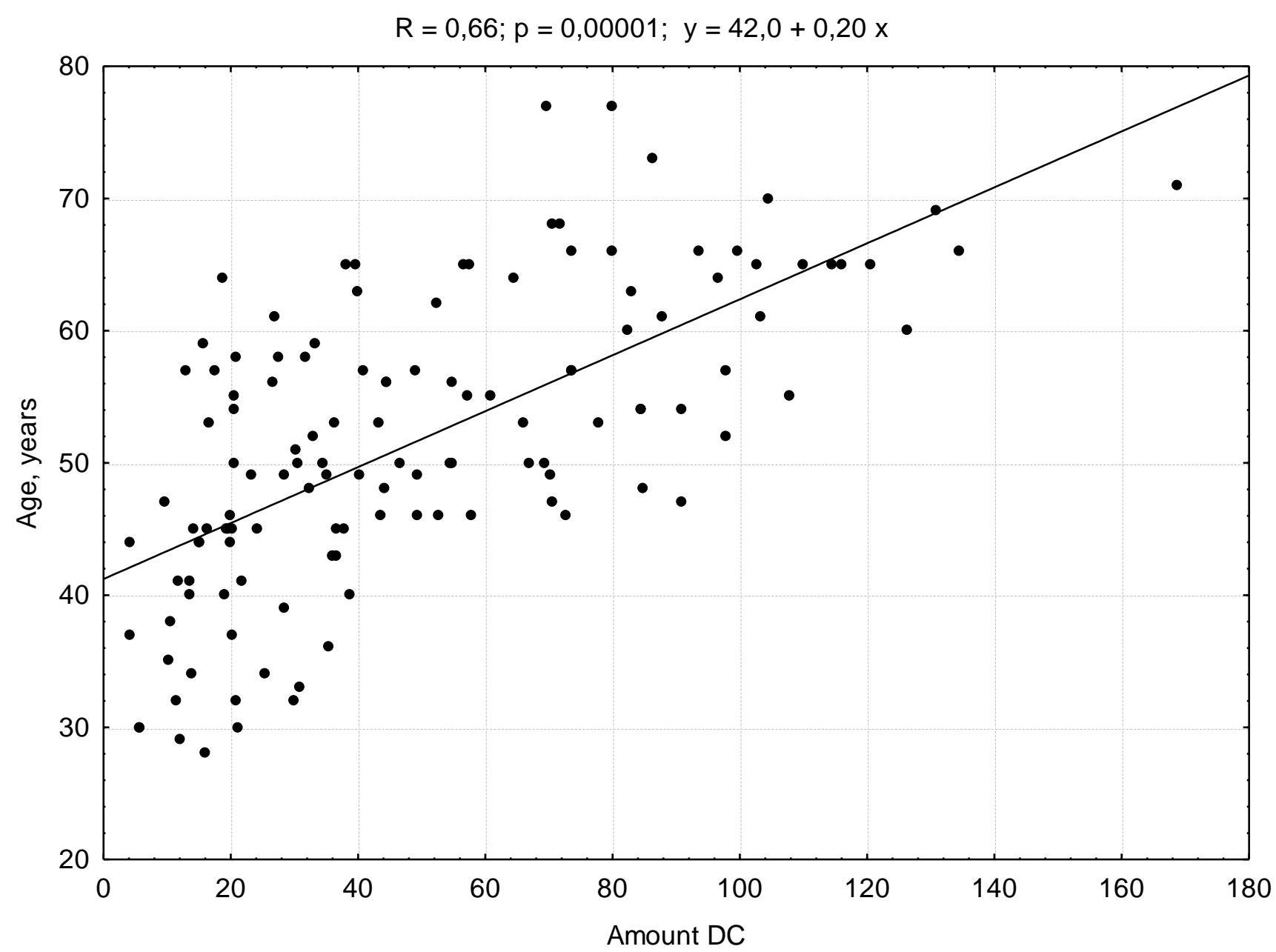

Figure 1.The graph of link between the amount of DC and the chronological age of the surveyed people

As you can see from the graph, the direct of the linear regression crosses the $\mathrm{Y}$ axis (age) at the point of 42 years. Therefore, as our BA formula suggests, even if a person does not have any indicator of aging, the calculation of BA will give a value of 42 years. It follows that the received formula is correct to be applied for the people who are older than this age.

According to the received formula, the BA of the surveyed people was calculated. On the figure 2 it's shown the ratio of the chronological age to BA of these people. 


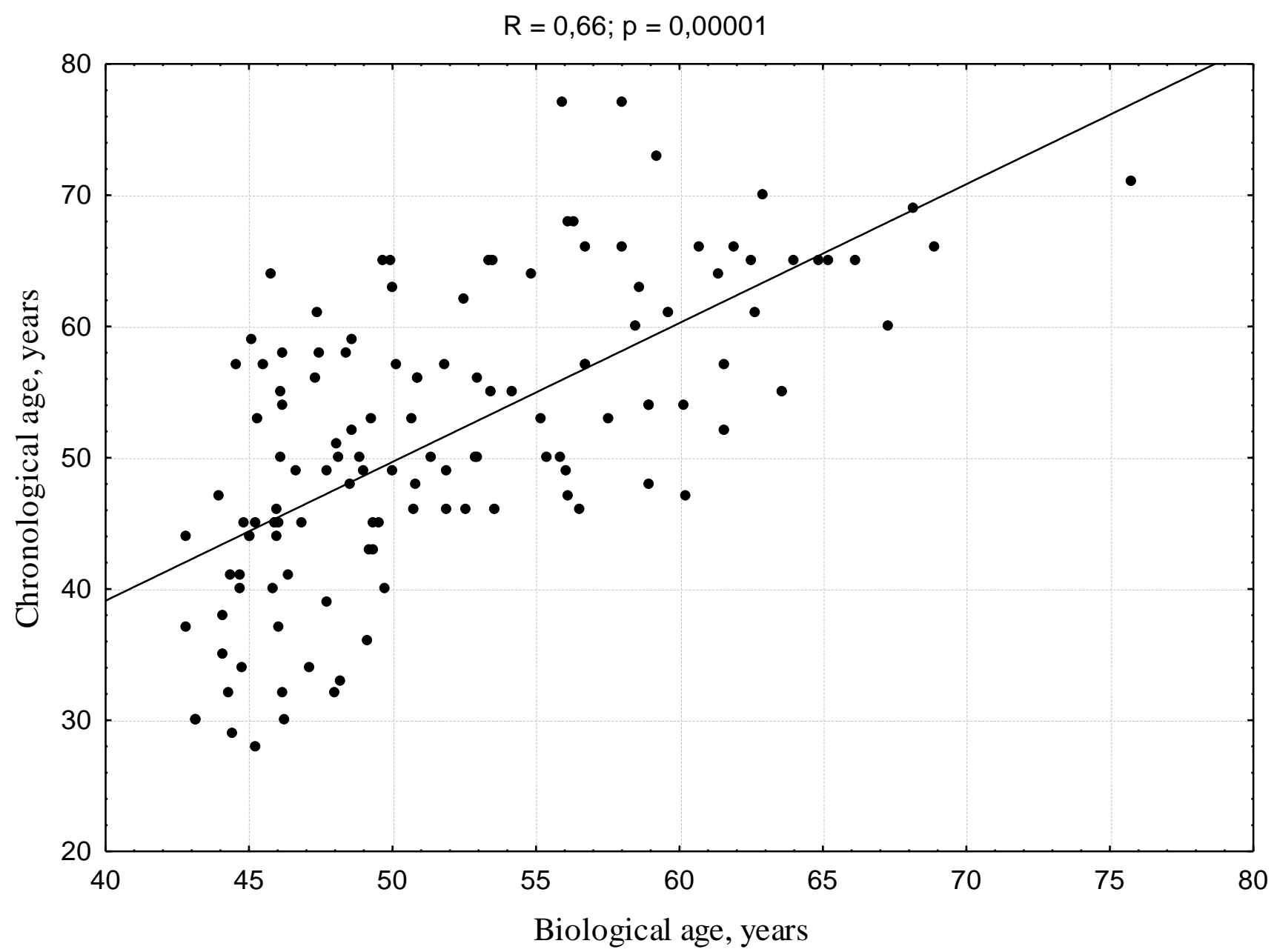

Figure 2. The ratio of the chronological and biological age of the surveyed people.

The difference between BA and the chronological age of each person was calculated. The average absolute difference was 6.6 years and the standard error was 8.3 years.

This is a good result for such a simple method. Usually even complex instrumental methods give an error of 5-10 years. The exceeding of BA over the chronological age by more than 10 years can be chosen as a diagnostic criteria of accelerated aging. In our study, such people made up $10.9 \%$. Verification of the received BA calculation formula will be carried out in the future as the data is set.

Thus, our express method of diagnosing of accelerated aging is simple, easy and accessible of use in outpatient and inpatient conditions. It does not require the use of instrumental and laboratory research and takes not much time. This method can be used to pre-selection of the patients with a risk of accelerated aging for further in-depth research. Implementation of the proposed method will allow not only to identify people with the risk of premature aging, but also to pre-evaluate the level of health, to predict the risk of pathology, to assess the effectiveness of treatment and prevention and rehabilitation activities. The proposed method can be used in scientific medical, sports institutions, medical and preventive institutions and clinical diagnostic centers.

Author Contributions: All authors participated equally in writing this commentary.

Conflicts of Interest: The authors declare no conflict of interest.

Information about Authors:

Anatoly V. Pisaruk - DSc (Medicine), Head of the Laboratory for Mathematical Modeling of Aging Processes; https://orcid.org/0000-00015522-0172

Valeri B. Shatilo - DSc (Medicine), Prof., Leading Researcher of the Department of Clinical Physiology and Pathology of Internal Organs, Deputy Director for Research; https://orcid.org/0000-0001-6420-000X

Ivanna A. Antonyuk-Shcheglova - DSc (Medicine), Leading Researcher of the Department of Clinical Physiology and Pathology of Internal Organs; https://orcid.org/0000-0002-6122-8266

Svitlana S. Naskalova - PhD (Medicine), Senior Researcher of the Department of Clinical Physiology and Pathology of Internal Organs; https://orcid.org/0000-0001-9518-2633 


\section{References}

1. Crowson, C.S.; Therneau, T.M.; Davis J.M.; Roger V.; Matteson, E.; Gabriel, Sh. Brief report: accelerated aging influences cardiovascular disease risk in rheumatoid arthritis. Arthritis Rheum 2013, 65, 10, 25622566. doi: 10.1002/art.38071

2. Lakatta, E.G. So! What's aging? Is cardiovascular aging a disease? J Mol Cell Cardiol 2015, 83. 1-7. doi: 10.1016\%2Fj.yjmcc.2015.04.005

3. Salthouse, T.A. Aging and measures of processing speed. Biol Psychol 2000, 54, 35-54. doi: 10.1016/s03010511(00)00052-1

4. Steffener, J.; Habeck, C.; O'Shea, D; Razlighi, Q.; Bherer, L.; Stern, Ya. Differences between chronological and brain age are related to education and self-reported physical activity. Neurobiology of Aging $2016,40$. doi: 10.1016/j.neurobiolaging.2016.01.014

5. Cevenini, E.; Invidia, L.; Lescai, F.S.; Salvioli, S.; Tieri, P. et al. Human models of aging and longevity. Expert Opinion on Biological Therapy 2015, 8, 9, 1393-1405. doi: 10.1517/14712598.8.9.1393

6. Cardoso, A.L.; Fernandes, A.; Aguilar-Pimentel, J.A.; de Angelis, M.H.; J. R. Guedes et al. Towards frailty biomarkers: Candidates from genes and pathways regulated in aging and age-related diseases // Ageing Res Rev. 2018. Nov. 47. 214-277. https://doi.org/10.1016/j.arr.2018.07.004

7. Belsky, D.W.; Caspic, A.; Houtsc, R.; Cohen, H.J.; Corcoran, D.L. et al. Quantification of biological aging in young adults. PNAS 2015, 27. doi: 10.1073/pnas.1506264112

8. Burkle, A.; Moreno-Villanueva, M.; Bernhard, J.; Blasco, M.; Zondag, G. et al. MARK-AGE biomarkers of ageing. Mechanisms Ageing Dev 2015. doi: 10.1016/j.mad.2015.03.006

9. Xia, X.; Chen, W.; McDermott, J.; Han, J.-D.J. Molecular and phenotypic biomarkers of aging: Version 1. F1000Res 2017, 6. doi: 10.12688\%2Ff1000research.10692.1

10. Sebastiani, P.; Bae, H.; Sun, F.X.; Andersen, S.L.; Daw, E.W., et al. Metaanalysis of genetic variants associated with human exceptional longevity. Aging (Albany NY) 2013, 5, 9, 653-659. doi: 10.18632/aging.100594

11. Caballero, F.F.; Soulis, G.; Engchuan, W.; Sanchez-Niubo, A.; Arndt, H. et al. Advanced analytical methodologies for measuring healthy ageing and its determinants, using factor analysis and machine learning techniques: the ATHLOS project. Sci Rep 2017, 7, 43955. doi: 10.1038/srep43955.

12. Moreno-Villanueva, M.; Capri, M.; Breusingc, N.; Siepelmeyer, A.; Sevini, F. et al. MARK-AGE standard operating procedures (SOPs): A successful effort. Mech. Ageing Dev. 2015. doi: 10.1016/j.mad.2015.03.007 